\title{
Die fossilen Böden im Lößprofil Wallertheim (Rheinhessisches Tafel- und Hügelland) ${ }^{1}$ )
}

\author{
Von Hartmut Leser, Hannover \\ Mit 4 Abbildungen
}

$\mathrm{Z}$ us a mmen $\mathrm{f}$ assung. Das Profil Wallertheim/Rheinhessen (Kreis Alzey) wurde in den letzten Jahren mehrfach sedimentologisch und bodengeographisch untersucht. An der Basis des Profils wurden dunkelbraune Bodenhorizonte beobachtet, deren Einordnung sowohl in das Eem als auch in das Altwürm möglich schien. Neue Untersuchungen haben ergeben, daß die Lösse in das Jung-, Mittel- und Altwürm gehören. Die fossilen Böden an der Profilbasis sind mit den altwürmzeitlichen „Mosbacher Humuszonen" gleichzusetzen.

$\mathrm{Sum}$ mary. The soil-profile of Wallertheim/Rheinhessen (near Alzey) was investigated in recent years several times sedimentologically and pedologically. At the basis of this profile dark brown soil-horizons have been observed; the stratigraphical order and age of which were originally considered to be both Eem and Early Wuerm. New investigations have shown that the loesses belong to Late Wuerm, Middle Wuerm, and Early Wuerm. The fossile soils of the basis of the profile are comparable to the "Mosbacher Humuszonen" of Early Wuerm age.

\section{Problemstellung}

Nach dem Auffinden einer altpaläolithischen Jagdstelle bei Wallertheim durch Schmidtgen \& Wagner (1929/1931), wurde das Lößprofil in der Ziegelei Schick mehrfach untersucht und publiziert. Neben den Neuuntersuchungen durch Fauler (1938) und Leser (1967) wird bei Schmidtgen \& Wagner (1931), Woldstedt (1958), Falke (1960) und Semmel $(1968 a, 1969)$ darüber referiert. Dem Vorkommen des Tuffbändchens sind Arbeiten von Frechen (1959a, b) und Schönhals (1959a) gewidmet. Im Jahre 1964 äußerten sich dazu in einer mündlichen Diskussion auch ZaKoseK und Semmel (vgl. LeSER 1967, S. 158).

Ein Vergleich der Stratigraphie in den zitierten Arbeiten wird dadurch erschwert, daß jede Profilaufnahme in einem anderen Abbaustadium erfolgte, die Abbauwände demzufolge immer eine andere Orientierung aufwiesen. Gewiß wäre es leicht, die von den einzelnen Autoren ausgegliederten Horizonte miteinander zu vergleichen. Die dann auftretenden Übereinstimmungen würden aber keine einwandfreie Parallelisierung bedeuten.

Seit meiner Untersuchung im Jahre 1963 (LESER 1967) suchte ich das Profil mehrfach auf, um im Zuge des fortschreitenden Abbaus eine Übersicht über die Lage und Ausbildung der Bodenhorizonte zu behalten. Vor allem die im Gespräch durch ZAKOSEK und SEMMEL geäußerten Gedanken veranlaßten mich, das Problem weiterzuverfolgen. Außerdem erschien mir nach weiteren neun Besuchen bis August 1969 in der Grube eine Sichtung der bislang gegebenen Einstufungen der Böden angezeigt. In der schon zitierten Arbeit (LESER 1967) konnte diese Diskussion aus verschiedenen Gründen nicht durchgeführt werden.

Es muß also überprüft werden, welche der vorgenommenen Datierungen unter den gegenwärtigen Aufschlußverhältnisse noch aufrechterhalten werden können, wenn man die älteren Profile aus den vergangenen vier Jahrzehnten mitberücksichtigt. Mir liegt dabei die Absicht fern, eine endgültige Einordnung der Böden von Wallertheim vornehmen zu wollen. Diese ist schon deswegen nicht möglich, weil mehrere Autoren am Profil arbeiten und ihre Untersuchungen derzeitig z. T. noch laufen.

1) Herrn Professor Dr. A. Semmel möchte ich für die Aussprache am Aufschluß herzlich danken. 


\section{Grundlagen}

Der Profilbeschreibung wird eine Schilderung der Landschaft und eine Charakterisierung des heutigen Aufschlusses vorangestellt, um Möglichkeiten zur Einschätzung der Morphodynamik dieses Raumes zu geben.

\subsection{L a n d c haf t}

Wallertheim liegt am Wiesbach. Das muldenförmige Tal ist ein Teil des Hügellandes zwischen Wies- und Appelbach. Das Gebiet baut sich aus Schleichsanden (Ob. Mittel-Oligozän) auf, die höchsten Erhebungen, Schwarzen-Berg und Kuhhimmel, aus Cyrenenmergel und Süßwasserschichten (Unt. und Ob. Ober-Oligozän). Die Ostflanke dieses Hügellandes trägt eine Lößdecke, die bis an den Wiesbach hinabzieht. Gegliedert wird diese Abdachung durch Dellen und Trockentälchen. An einzelnen Stellen des Hanges kommen Reste pleistozäner Wiesbachterrassen vor (WAGNER 1935).

Auf der anderen Talseite tritt das Hügelland ebenfalls wieder auf (LESER 1965, 1966). Hier jedoch bildet es den Sockelteil der Nordwest- und Westrheinhessischen Plateaus, die durch zahlreiche Dellen und Tälchen stark aufgelöst sind. Stufenrandbuchten (Vendersheim, Sulzheim, Rommersheim) werden von Ausliegern oder Zeugenbergen (Wißberg) abgelöst. Die Plateaus tragen über den Süßwasserschichten eine Kalkdecke (Miozän). Meist handelt es sich um die Inflata-Schichten, weniger um die Cerithien-Schichten. Ebenso wie die unteren Teile des Hügellandes um den Wiesbach tragen auch die Plateaus eine Lößdecke, die auf den Hochflächen jedoch meist pliozäne Sande überlagert. Lößfrei sind die Gehänge der Plateaus im Sand- und Mergelstockwerk (Oligozän). In diesem Bereich spielen sich lebhafte Rutschvorgänge ab, die auch während des Pleistozäns wirksam waren und dabei die Lösse größtenteils entfernten oder wenigstens umlagerten, wie es in vielen rheinhessischen Profilen zum Ausdruck kommt. Wie jenseits des Wiesbaches kommen auch auf der Ostseite Reste pleistozäner Wiesbach-Terrassen vor. Der Mangel an Aufschlüssen verbietet jedoch deren Einordnung in das Schema der Pleistozängliederung.

\subsection{Lage und Beschaffenheit des Profils}

Die Grube befindet sich südlich Wallertheim. Der Ort liegt wenige Meter über dem Wiesbach am untersten Ende eines langen Gleithanges, der vom Schwarzen-Berg zum Wiesbachknie herunterzieht. Wie aus den Terrassenresten zu beiden Seiten des Baches hervorgeht, muß die heutige Fließrichtung mindestens seit dem Mittelpleistozän innegehalten worden sein. Nur so ist die große Stufenrandbucht zwischen Rommersheim-SulzheimVendersheim und dem Wißberg sowie das Vorkommen von Wiesbachschottern unterschiedlicher Höhenlage in der Bucht zu deuten. Die niedrigeren Formen auf der Westseite des Wiesbaches und der Abfall der Plateaus auf der Gegenseite zeigen, daß es sich hierbei um einen großen, mehrfach gegliederten Gleit- und Prallhang handelt. Im Zuge der Tieferlegung des Wiesbaches erfolgte dann eine weitere Untergliederung dieser Großform.

Das Lößprofil Wallertheim wurde zuletzt an der Grubenwestwand aufgenommen. Gegenüber der Aufnahme von Schmidtgen \& WAGNer (1929) bedeutet das beachtliche Verschiebungen des Aufschlusses. Im Jahre 1963 war die Westwand fast in der gleichen Position wie 1966/1967 aufgeschlossen, nur war das fossile Bodensediment gerade an seiner Oberfläche angeschnitten, das heute eventuell als Durchmischungszone von B- und CMaterial gedeutet werden muß; dies war jedoch seinerzeit nicht einwandfrei zu erkennen. Inzwischen wurde die Grubensohle tiefer gelegt, wobei aber große Teile der Jüngeren Mittelterrasse des Wiesbaches zerstört worden sind. Reste waren aber bis 1967 noch vorhanden, so daß der Anschluß an die Hangendsedimente hergestellt werden konnte. Der Terrassenkörper ist jedoch zerstört. 
An der gesamten Westwand war (mit Unterbrechungen) von 1967 bis 1969 ein zweigegliederter, schwarzbrauner bzw. dunkelbrauner fossiler Boden sichtbar. Sein unterer Abschnitt ist vom oberen durch eine helle Lößschicht getrennt, die aber nach Norden hin auskeilt, so daß sich beide Bodenhorizonte vereinigen. Insgesamt sprechen die Lagerungsverhältnisse für eine Mulde (Abb. 3). An der Südostwand setzt sich der obere Boden fort. Der Unterteil ist jedoch noch nicht aufgeschlossen. Grabungen ergaben aber, daß hier die

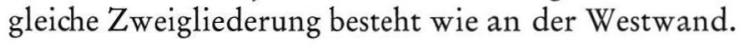

Sowohl an der West- als auch an der Südostwand sowie an der heute nicht mehr abgebauten Nordwand ist $200 \mathrm{~cm}$ unter der rezenten Landoberfläche das ca. 2 bis $3 \mathrm{~cm}$ mächtige Tuffbändchen aufgeschlossen, das lange Zeit dem Kärlicher Ausbläser zugeordnet wurde (Frechen 1959a, b, 1962, Schönhals 1959a, LeSER 1967). Seine stratigraphische Position veranlaßte Semmel, bei diesem oder bei stratigraphisch gleichen Tuffbändchen vorläufig vom „Eltviller Tuff“ zu sprechen [dazu Diskussionsbemerkungen von SEMmEL (1968a), außerdem in dem Aufsatz Semmel (1967)]. Die gleiche Tiefe wurde von SchmidtGEN \& WAGNER (1929) festgestellt, wenngleich man seinerzeit im Tuffbändchen einen „Verlehmungsstreifen im Jüngeren Löß II“ sehen wollte: „Nicht unerwähnt darf bleiben, daß auch dieser Jüngere Löß II nicht eine ununterbrochene Lößbildung darstellt. Ziemlich genau $2 \mathrm{~m}$ unter der Ackerkrume zieht sich durch das ganze Grubengebiet ein verlehmter Lößstreifen von etwa $2 \mathrm{~cm}$ Stärke, der auf eine kurze Klimaschwankung während dieser Lößbildung hinweist“ (Schmidtgen \& Wagner 1929). Auch in der durch Schmidtgen angeregten Arbeit Faulers (1938) wird das Bändchen als Verlehmungszone eingestuft. Siehe dazu auch die Bemerkungen von Schönhals (1959a). - Neuerlich wurde das Bändchen von Andres und Semmel untersucht.

\section{Profilbeschreibung}

Für die Gliederung des Profils kommt neben den fossilen Böden auch den Terrassenschottern entscheidende Bedeutung zu. Sie grenzen das Profil gegen das Liegende ab und stellen eine Datierungshilfe dar. Außerdem läßt die Terrasse wichtige Schlüsse hinsichtlich des Klimas zu.

Schon Schmidtgen \& Wagner $(1929,1931)$ sowie Fauler $(1938)$ erkannten den Wert der Terrassenablagerungen. In der Terrasse wurden durch Schmidtgen \& Wagner Steinwerkzeuge eiszeitlicher Jäger gefunden, ebenso eine Fauna. Die Datierungen der Autoren unterscheiden sich voneinander. Während die paläolithischen Werkzeuge von den erstgenannten Autoren in den Vorstoß der Würm-Kaltzeit gestellt werden, sind die Funde nach FAUler rißglazialen Alters.

Von den früheren Bearbeitern wurden die Sedimentanalysen zwar auch schon angewandt, jedoch bleiben dabei geomorphologische und landschaftsökologische Aspekte im Hintergrund. Dieser Vorwurf wurde auch gegenüber WAGNER bei der DEUQUA-Exkursion 1951 nach Wallertheim erhoben. ${ }^{2}$ )

\subsection{Wiesbachterrassen}

Die Wiesbachterrassen in der Grube Wallertheim erfuhren durch die einzelnen Autoren ganz unterschiedliche Einstufungen. Schmidtgen \& WAgneR (1929) stellten für den Wiesbach folgende Terrassengliederung auf:

2) Mitteilung durch Herrn Professor Dr. Dr. h. c. C. Troll, der mir seine Notizen von der DEUQUA-Exkursion 1951 freundlicherweise zur Verfügung stellte. 


$\begin{array}{lrrl} & \text { Höhenlage } & \text { Mächtigkeiten } & \\ \text { Niederterrasse } & 3,5-4 \mathrm{~m} & 1-1,5 \mathrm{~m} & \text { 2. Vorstoß der Würmeiszeit } \\ \text { Talwegterrasse } & 8 \mathrm{~m} & 0,8-1,2 \mathrm{~m} & \text { 1. Vorstoß der Würmeiszeit } \\ \text { Hochterrasse } & 30-35 \mathrm{~m} & {[0,5-1,2] \mathrm{m}} & \text { Rißeiszeitgruppe } \\ \text { Hauptterrasse } & 80 \mathrm{~m} & 10,0 \mathrm{~m} & \text { Mindel- oder Günzeiszeitgruppe }\end{array}$

weil neuere Untersuchungen ergeben haben, daß Kärlich nicht den Herkunftsort des Tuffes darstellt, den Semmel (1967) jetzt als „Eltviller Tuff“ bezeichnet hat. Immerhin läßt er doch eine Trennung der Naßböden zu.

Mächtigkeiten wurden von Schmidtgen \& WAgner (1929) für die „Rißeiszeitgruppe“ nicht angegeben. (Zahlen nach Leser 1967. Diese Terrasse wurde aber hier als Altere Mittelterrasse des Wiesbaches eingestuft.) FAULER (1938), der sich nur zur Nieder- und Talwegterrasse äußerte, übernahm die Einstufungen der beiden Autoren und machte sie zur Grundlage seiner Datierungen.

Zunächst wären die Aufschluß- und Lagerungsverhältnisse der Terrasse zu beschreiben, wie sie bis kurz vor der Zerstörung des Schotterpaketes zu beobachten gewesen sind. Es muß dann geprüft werden, ob diese Terrasse mit den Terrassenkörpern, wie sie von Schmidtgen \& Wagner (1929) bzw. Fauler (1938) beschrieben wurden, identisch ist. Außerdem wäre die Stellung der Niederterrasse dieser Autoren zu den hangenden Lössen und ihren fossilen Böden zu klären.

Die Terrasse war im Sommer 1963 vor der Südsüdwestwand in einem etwa W-E verlaufenden Schnitt aufgeschlossen. Aus dem 1967 publizierten Profil (Leser 1967) geht hervor, daß der um $100 \mathrm{~cm}$ mächtige Schotterkörper in sich gestuft ist und daß seine Mächtigkeit schwankt. Die Schotterlage zeigte aber, daß im Westteil des Aufschlusses die Obergrenze der Terrasse zu suchen ist bei $128 \mathrm{~m} \mathrm{NN}$, während die Untergrenze an der Ostflanke noch nicht erreicht war, jedoch unter $127 \mathrm{~m}$ NN liegen mußte. Das bedeutet eine Lage von unter $5,5 \mathrm{~m}$ über dem heutigen Wiesbach, der südlich von Wallertheim - auf der Höhe der Grube - im Niveau von 122,5 m NN fließt.

Im Vergleich zu Schmidtgen \& WaGner (1929) ergeben sich folgende Konsequenzen: Die auf einer Breite von $40 \mathrm{~m}$ aufgeschlossene Terrasse liegt niveaumäßig zwischen den von o. a. Autoren angegebenen Werten für die Niederterrasse (Unterkante bei $3,5-4 \mathrm{~m}$ ) und die untere Mittelterrasse (Unterkante bei $8 \mathrm{~m}$ ). In der Skizze, die Schmidtgen \& Wagners Arbeit beigefügt ist, wird eine Niederterrasse als Grubensohle vorgeführt, deren Oberfläche bei $124 \mathrm{~m}$ NN liegt (= 1,5-2-2,5 $\mathrm{m}$ über dem Wiesbach).

Bei der 8-m-Terrasse Schmidtgen \& Wagners (1929) und meiner 5-6-m-Terrasse muß es sich jedoch um die gleichen Bildungen handeln, wie aus dem Vergleich des Geröll- und Fossilinhaltes hervorgeht. Eine morphometrische Schotteranalyse (LESER 1967) erbrachte - neben dem Auffinden von kryoturbaten Verwürgungen und offenbar frostgesprengten Geröllen —, daß es sich bei der Terrasse um eine kaltzeitliche Bildung handeln muß. Nicht mehr aufgefunden werden konnte die $2 \mathrm{~m}$ über dem Wiesbach liegende Niederterrasse, deren Oberfläche von den o. a. Autoren auf $124 \mathrm{~m} \mathrm{NN}$ angesetzt wurde. Da die von mir aufgesundene Terrasse erheblich über der Talsohle liegt, handelt es sich vermutlich um eine Mittelterrasse. Für die Einstufung der Decksedimente ist diese Terrasse von Bedeutung.

\subsection{Lößstratigraphie}

Wie eine neuerliche Untersuchung des Lößprofils Wallertheim ergeben hat, scheint die Untergliederung des Alt- und Mittelwürmlösses einer Korrektur zu bedürfen. Ich nahm an, daß die Grenze an dem Südsüdwestwand-Profil bei $520 \mathrm{~cm}$ unter der rezenten Landoberfläche liegt, wo ein Geröllhorizont geringer Mächtigkeit eine Erosionsdiskordanz andeutet. Das Zurücktreten der Lößfraktion sowie der unterschiedliche Kalkgehalt oberhalb und unterhalb dieser Linie ließen neben dem anderen Charakter des Lösses hier einen Hiatus vermuten. Dieser liegt gewiß auch vor, doch darf seine pedogenetische und klimageographische-geomorphologische Bedeutung nicht überschätzt werden.

Bei Vergleich der beiden Profile, nämlich dem im Sommer 1963 an der Südsüdwestwand aufgenommenen und dem im Herbst 1966 bzw. Sommer 1967 an der Westwand beobachteten, sind folgende Schlüsse zu ziehen (auf die Gegebenheiten von 1969 mit den 
gegenüber 1967 vergleichsweise geringen Änderungen der Aufschlußverhältnisse wird noch weiter unten eingegangen): Trotz der unterschiedlichen Mächtigkeit ist zu vermuten, daß beide Profile im wesentlichen einander entsprechen. Als Kennzeichen lassen sich der Charakter und die Ausbildung der Lösse sowie der fossilen Böden heranziehen. Ich gehe davon aus, daß es sich bei den Böden um gleichaltrige Bildungen handelt, weil sich die stratigraphischen Positionen entsprechen. Der Aufbau des Lößprofils an der Südsüdwestwand (Oberkante des Profils bei $135 \mathrm{~m} \mathrm{NN}$ ) wurde bereits in einer Publikation geschildert, so daß sich eine Wiederholung an dieser Stelle erübrigt (LESER 1967; die Horizontbeschreibungen wurden in dieser Publikation leicht gekürzt). Im Sommer 1967 erschien nun das neuerdings von der Westwand beschriebene Profil auch an der Südsüdwestwand. Während es 1969 in der letztgenannten Position mehr oder weniger gleich gut aufgeschlossen war, konnte es an der zerstörten Westwand nur noch punkthaft verfolgt werden.

Das Profil baut sich wie folgt auf: Der Boden an der rezenten Landoberfläche ist auf einem Löß gebildet, dessen Basisteil vom Band des „Eltviller Tuffs" markiert ist. Darunter folgt weiterhin Löß, der etwa bei $380-420 \mathrm{~cm}$ durch eine schwache Bodenbildung gekennzeichnet war. Danach folgt wiederum Löß, der dann deutlich gegen den hier im Detail zu schildernden Profilteil (= Profil II) angesetzt ist (Abb. 1):

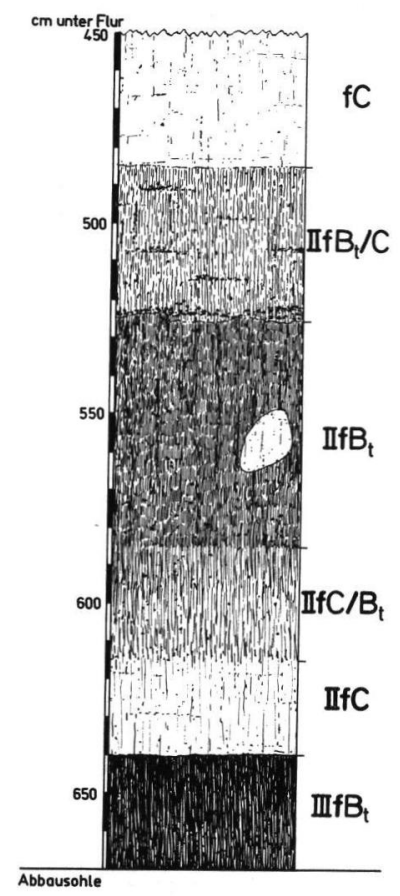

Abb. 1. Mosbacher Humuszonen im Profil Wallertheim/Rheinhessen im Jahre 1967.

1. $\left.485 \mathrm{~cm} \mathrm{fC}^{3}\right) \quad$ Heller, gelblich-brauner Löß (10 YR 7/4 - 10 YR 6/4). Schluffig-toniger Lehm bis lehmiger Ton. Porös. Kalkröhrchen, Wurzelspuren, kleine Manganflecken.

2. 485-525 cm IIfB $/ \mathrm{t}$ C Heller, gelblich-brauner Löß (10 YR 6/4). Deutlich dunkler als fCHorizont. Lehmiger Ton. Nach oben deutlich mit scharfer Linie abgegrenzt, stellenweise jedoch auch diffuser Übergang. Horizont sehr fest.

3) Die den Horizontsymbolen vorangestellten römischen Ziffern sind nur für den $\mathrm{h}$ i e $\mathrm{r}$ beschriebenen Profilteil gültig und der besseren Unterscheidung halber eingesetzt. Sie bedeuten keine Parallelisierung mit den Horizonten des in meiner Arbeit von 1967 geschilderten Profils aus Wallertheim. 
3. 525-585 cm $\operatorname{IIfB}_{\mathrm{t}}{ }^{4}$ ) Obergrenze scharf und linienhaft. Von Kiesbändchen umgeben, jedoch nur oberhalb. Grenze sehr deutlich, jedoch stark auf- und abschwingend. Kiesbändchen z. T. mit kleinen, gelbbraunen Kalkkonkretionen versetzt. Dunkler, gelblich-brauner lehmiger Ton (10 YR 5/4). Relativ fest. Subpolyedergefüge. Nach unten zu schalten sich bräunliche, hellere Lößflecken ein (bis $0,5 \mathrm{~cm}^{2}$ groß). Werden aber erst im IIfC/ $\mathrm{B}_{\mathrm{t}}$ zahlreicher. Wurzelspuren. Kalkröhrchen. Im Horizont: große Krotowine $(20 \mathrm{mal} 30 \mathrm{~cm}$, oval). An der Untergrenze, ebenso an der von IIfC/ $/ \mathrm{B}_{t}$, Frostspalten und Eiskeile.

4. 585-615 cm IIfC/B $\mathrm{B}_{\mathrm{t}}$ Dunkler, gelblich-brauner lehmiger Ton durch helleren, gelblich-braunen lehmigen Ton (10 YR 6/4) abgelöst. Helle Lößflecken werden zahlreicher und gehen an Untergrenze in Löß des IIfC-Horizontes über. Kompakt. Zahlreiche Wurzelspuren mit schwarzbrauner Auskleidung. Im Horizont und an der Untergrenze (bis in IIfC-Horizont hineinreichend): Eiskeile und Frostspalten.

5. 615-640 cm IIfC Heller, blaßbrauner poröser Löß (10 YR 7/4). Dunkles IIfB t $_{\text {-Material }}$ tritt kaum noch auf oder nur sehr blaß (Obergrenze!). Wurzelröhrchen dunkel ausgekleidet.

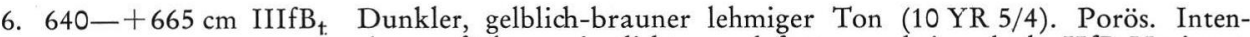
siver gefärbt sowie dichter und fester erscheinend als IIfB-Horizont. Subpolyeder- bis schwach ausgebildetes Polyedergefüge. Weiße Kalkröhrchen und punktförmige Kalkausscheidungen.

Der makroskopische Vergleich von den Unterteilen der beiden Profile erbringt, daß die Horizonte $\mathrm{f} 3 \mathrm{~A}(\mathrm{~B})-(\mathrm{B}) \mathrm{C}$ und $\mathrm{IIfB}_{t} / \mathrm{C}$ offenbar nicht $\mathrm{zu}$ parallelisieren sind. Beide besitzen jedoch die gleiche Farbe (10 YR 6/4), sind sehr fest und führen Kiesstücke oder Kiesbändchen. Die Korngrößenanalysen (Abb. 2) zeigen folgende Werte 5):

\begin{tabular}{|c|c|c|c|c|c|c|c|c|c|c|c|c|}
\hline & & I & II & III & IV & $\mathrm{V}$ & VI & VII & VIII & IX & $\begin{array}{l}\text { Feinheits- } \\
\text { grad }\end{array}$ & $-\mathrm{CaCO}_{3}$ \\
\hline \multirow{2}{*}{ Profil I } & $\mathrm{f}_{3} \mathrm{~A}(\mathrm{~B})$ & 24.6 & 6.4 & 20.4 & 40.0 & 3.9 & 2.7 & 1.3 & 0.4 & 0.3 & 75.1 & $23.8 \%$ \\
\hline & $\mathrm{f}_{3} \mathrm{~B}(\mathrm{C})$ & 21.1 & 6.0 & 20.6 & 36.5 & 6.3 & 4.2 & 1.8 & 0.5 & 0.0 & 69.8 & $33.6 \%$ \\
\hline \multirow[t]{2}{*}{ Profil II } & $\begin{array}{l}\text { IIfB }_{t} / \mathrm{C} \\
\text { (oben) }\end{array}$ & 32.1 & 5.3 & 14.0 & 27.3 & 14.4 & 4.2 & 1.9 & 0.4 & 0.4 & 68.9 & $29.3 \%$ \\
\hline & $\operatorname{IIfB}_{t} / \mathrm{C}$ & 31.5 & 6.1 & 12.5 & 28.4 & 13.5 & 4.1 & 2.7 & 1.0 & 0.2 & 68.4 & $26.6 \%$ \\
\hline
\end{tabular}

Die hangenden C-Lösse der beiden Horizonte besitzen folgende Verteilung der Korngrößen, die - ebenso wie der Kalkgehalt - deutliche Übereinstimmungen aufweisen (Abb. 2):

\begin{tabular}{lllllllllllll} 
& & & & & & & & \multicolumn{2}{c}{ Feinheits- } \\
& & I & II & III & IV & V & VI & VII & VIII & IX & grad & $\mathrm{CaCO}_{3}$ \\
\hline Profil I & f $_{2}$ C & $\mathbf{2 1 . 4}$ & $\mathbf{5 . 5}$ & $\mathbf{2 0 . 9}$ & $\mathbf{4 3 . 6}$ & 3.8 & 3.3 & 1.0 & 0.4 & 0.1 & 73.2 & $\mathbf{2 2 . 3} \%$ \\
Profil II & fC (oben) & $\mathbf{2 2 . 2}$ & $\mathbf{4 . 7}$ & $\mathbf{2 0 . 0}$ & $\mathbf{4 0 . 6}$ & 9.2 & 1.8 & 1.1 & 0.2 & 0.2 & 67.9 & $\mathbf{2 2 . 6} \%$ \\
& fC (unten) & 31.5 & 5.3 & 15.9 & $\mathbf{3 0 . 4}$ & 7.9 & 5.3 & 3.0 & 0.5 & 0.2 & 69.1 & $\mathbf{2 8 . 2 \%}$
\end{tabular}

4) Das Horizontsymbol $B_{t}$ scheint für die 1966 in Wallertheim aufgeschlossenen und diesen Ausführungen zugrunde gelegten "Mosbacher Humuszonen“ angemessen. Ursprünglich handelte es sich vielleicht um schwarzerdeartige Steppenböden (s. a. SEmmel 1968b). Die Bt-Merkmale, die SchöNhals et al. (1964) für die letztinterglaziale Parabraunerde angegeben haben, treffen auch auf die beiden in Wallertheim beobachteten Humuszonen zu. Diese Merkmale waren ja zunächst auch eine der Ursachen, den 1963 in Wallertheim gefundenen Bodenrest ins Eem zu stellen.

5) Einteilung der Fraktionen in Korngrößenklassen: $I=<0,002 \mathrm{~mm}$, II $=0,006-0,002 \mathrm{~mm}$, III $=0,02-0,006 \mathrm{~mm}$, IV $=0,06-0,02 \mathrm{~mm}, \mathrm{~V}=0,12-0,06 \mathrm{~mm}, \mathrm{VI}=0,25-0,12 \mathrm{~mm}$, VII $=$ $0,5-0,25 \mathrm{~mm}, \mathrm{VIII}=1,0-0,5 \mathrm{~mm}, \mathrm{IX}=2,0-1,0 \mathrm{~mm}$. 


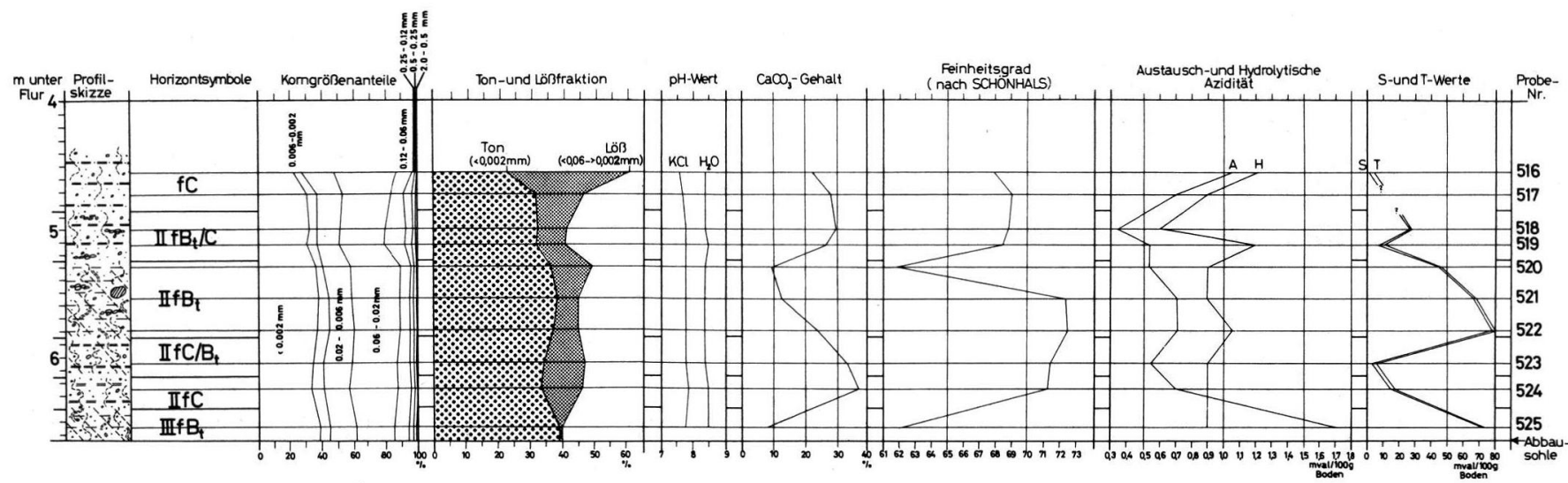

Abb. 2. Graphische Darstellung der Analysendaten des neu aufgeschlossenen Profilteils von Wallertheim mit den Mosbacher Humuszonen. 
Die Auswertung dieser Aufstellungen zeigt, daß zwischen den Böden bzw. Bodenresten der Profile I und II deutliche Unterschiede in den Korngrößenzusammensetzungen auftreten, bei den hangenden Lössen jedoch nicht. Auch die Kalkgehalte differieren. Nach dem Wechsel der Aufschlußverhältnisse darf vermutet werden, daß mit den $\mathrm{f}_{3}$-Horizonten des Profils I ein anderes Profilglied angeschnitten war, das sich nicht mit dem $\mathrm{IIfB}_{t} / \mathrm{C}$ von Profil II parallelisieren läßt. Semmel erwähnte mündlich, daß in Wallertheim zeitweise auch eine dritte Mosbacher Humuszone aufgeschlossen war. Möglicherwe is e handelt es sich bei den $\mathrm{f}_{3}$-Horizonten um Teile dieser, ohne daß damit ein Beweis für deren Existenz erbracht sein soll. Ich selbst fand sie in Wallertheim nach 1963 nicht. Die unterschiedliche Korngrößenzusammensetzung und die verschiedenen Kalkgehalte sind einwandfrei auf jeweils andere Ablagerungsbedingungen zurückzuführen.

\subsection{Fossile Böden}

Die rezente Landoberfläche wird von einem Steppenboden eingenommen, der aus den postglazialen Wärmezeiten stammt und aufgrund der für seine Erhaltung günstigen Klimabedingungen im Rheinhessischen Tafel- und Hügelland bis heute überdauern konnte. Man spricht daher von einem Reliktboden (r). Die Steppenböden des nördlichen Oberrheinischen Tieflandes wurden in neuerer Zeit grundlegend von ZAKOSEK (1962) untersucht. Dieser Steppenboden grenzt das Profil zeitlich nach oben hin ab, so wie die Mittelterrasse im Liegenden des Lößprofiles von Wallertheim eine Zeitmarke für das Präwürm darstellt.

Als nächster Horizont (Boden) folgt ein ca. $40 \mathrm{~cm}$ mächtiger Naßboden vom Typ des Frosttundrengleys. Er liegt oberhalb des Tuffbändchens und trat erstmals 1967 in Erscheinung. Unterhalb des „Eltviller Tuffs“ folgt ein Lößpaket, das möglicherweise einen schwach ausgebildeten Naßboden enthält. In seinem Liegenden tritt ein weiterer, ca. $60 \mathrm{~cm}$ mächtiger Naßboden auf. Der Boden wurde aufgrund der lößstratigraphischen Untersuchung als Repräsentant des Jung/Mittelwürm-Interstadials angesprochen (LESER 1967). Seine stratigraphische Position muß daher korrigiert werden, da er eindeutig in das Jungwürm zu gehören scheint. Er ließ sich in zahlreichen anderen Lößprofilen ebenfalls beobachten, war aber mit dem $f_{1} g$ von Wallertheim nicht gleichzusetzen. Die zeitliche Zuordnung von Sprendlingen I und II sowie in Monsheim dürfte richtig gewesen sein (LESER 1967). Bemerkenswert ist nämlich die folgende Beobachtung: Im Sommer 1967 war in der Grube Schnell in Sprendlingen (Straße nach St. Johann) der Boden ebenfalls unter dem Tuffbändchen aufgeschlossen. Er entsprach noch den Verhältnissen der Untersuchung von 1963, obwohl der Abbau weiter vorangeschritten war. In der Südecke der Grube setzte aber der graue Naßboden (Frosttundrengley) auf einer Breite von ca. $8-10 \mathrm{~m}$ aus und an seine Stelle trat ein dunkelbrauner (10 YR 6/4-5/4) Boden. Er ging seitlich allmählich aus dem ca. $85 \mathrm{~cm}$ mächtigen Frosttundrengley sehr rasch hervor und wurde gleichzeitig mächtiger (maximal ca. $110 \mathrm{~cm}$ ). Insgesamt ergaben sich für den Boden muldenförmige Ablagerungsverhältnisse. Seine Erhaltung verdankt er vermutlich anderen Grund- und Bodenwasserverhältnissen, die sich von denen seiner Umgebung unterschieden. Das seitliche Hervorgehen aus dem Frosttundrengley läßt dabei den Schluß zu, daß eventuell der gesamte Boden eine solche braune Farbe und ein Subpolyedergefüge bis krümeliges Gefüge aufwies und erst nachträglich die meisten Areale dieses Bodens überprägt wurden. Diese Deutung erscheint deswegen wahrscheinlich, weil der braune Lehm nach meinen Beobachtungen in Rheinhessen eigentlich den Ausnahmefall darstellt und Frosttundrengleye oder Braunerde reste in dieser stratigraphischen Position die Normalverhältnisse repräsentieren.

Im Sammelprofil für das Rhein-Main-Gebiet geben Schönhals, Rohdenburg \& Semmel (1964) als Abschluß des Mittelwürm den „Hahnstätter (Hainerberger) Boden“ an. In der stratigriphischen Position entspricht er den Frosttundrengleyen und Braunerde- 
resten aus dem Rheinhessischen Tafel- und Hügelland, die in das Jung/Mittelwürm-Interstadial eingestuft wurden. Diese Böden entsprechen wohl auch dem „Boden des Würm II/ III-Interstadials“, den Schönhals (1959a) für Wallertheim feststellte. Während im Rheinhessischen Tafel- und Hügelland bisher nur Frosttundrengleye und schwach ausgebildete Braunerdereste für das Jung/Mittelwürm-Interstadial festgestellt werden konnten, wurde mit dem oben beschriebenen Boden im Profil Sprendlingen erstmals eine in allen Merkmalen dem „Hahnstätter (Hainerberger) Boden“ äquivalente Bildung aufgefunden. Dazu Schönhals, Rohdenburg \& Semmel (1964): „Dort, wo der Boden der letzten Warmzeit nicht pseudovergleyt ist, hat er (der "Hahnstätter [Hainersberger] Boden“, Anm. v. Verf.) eine schwach rötlich getönte Farbe (7.5-10 YR 5/4), dort, wo der letztwarmzeitliche Boden eine Marmorierung aufweist, entspricht die Farbe 10 YR 5/4. Im ersten Falle zeichnet sich der Boden durch ein plattig überprägtes Krümelgefüge aus, im zweiten Fall besitzt er ein plattig-polyedrisches Gefüge mit dunklen Anflügen auf den Aggregatflächen. In beiden Böden sind im oberen Profilteil häufig Krotowinen zu beob, achten. Der Tongehalt beträgt 25-30\%.“

Für das Profil Wallertheim ergeben sich aus diesen Uberlegungen und Beobachtungen einige Konsequenzen. Unter dem zweiten Naßboden im Liegenden des „Eltviller Tuffs“ tritt zunächst Löß auf, den zahlreiche kryoturbat verwürgte Sandbändchen durchziehen. Auf diese Sandbändchen wurde schon hingewiesen (LESER 1967). Auch ANDrEs (1968) stellte sie im nördlichen Rheinhessen fest. Eingeschaltet in die Sandlagen ist ein schwaches mittelgraues Tuffband. Unter den Sandbändchen folgt ein ca. $50-60 \mathrm{~cm}$ mächtiger schwach humoser Boden, der den schon beschriebenen Naßböden sehr ähnlich sieht. Seine Mächtigkeit und seine stratigraphische Position lassen vermuten, daß er dem „Hahnstätter (Hainerberger) Boden “ von SchönHAls et al. (1964) entspricht, mithin möglicherweise in das Paudorf-Interstadial gehört. Bei Vergleich mit Sprendlingen (s.o.) liegt der Schluß sehr nahe, daß dieser Jung-Mittelwürm-Interstadialboden - auch aufgrund seiner stratigraphischen Einordnung durch ScHöNHALs (1959) - vermutlich dem „Hahnstätter (Hainerberger) Boden“ entspricht. Diese Vermutung wurde schon in der Arbeit von 1967 ausgesprochen (LESER 1967) ${ }^{6}$ ). Ich möchte diese Datierung auch weiter beibehalten, obwohl sich vielleicht auch eine Übereinstimmung mit den Merkmalen des "Gräselberger Bodens“ finden ließe. Den „Kirchberger (Gräselberger) Boden“ konnte ich im Rheinhessischen Tafel- und Hügelland, mit Ausnahme des Profils Monsheim (Südrheinhessen), nicht finden. Schönhals, Rohdenburg \& Semmel (1964) dazu: „Der ,Kirchberger (Gräselberger) Boden' besitzt in den außerhessischen Lößgebieten, soweit sie den Verfassern bekannt sind, kein sicheres Äquivalent. Es kann höchstens vermutet werden, daß er dem ,FWc-Boden“ BRUNNACKERs (u. a. 1958, S. 130) entspricht."

Bevor die Basis des Profils mit den mächtigen intensiv gefärbten Böden erreicht ist, tc noch ein weiterer Bodenrest auf. Es handelt sich um einen grau-bräunlichen Löß wechinder Mächtigkeit, den Semmel (1969) als „Nieder-Eschbacher Zone“ auszuscheiden vermag.

Der nächste einwandfrei zu beobachtende Boden neben dem reliktischen Steppenboden aus dem Klimaoptimum des Postglazials (ZAKoseK 1962) und dem Jung/MittelwürmInterstadialboden (entsprechend dem "Hahnstätter (Hainerberger) Boden“ von ScHöNhals, Rohdenburg \& Semmel (1964) sind die mächtigen dunkelbraunen Horizonte an der Basis des Lößprofiles von Wallertheim, die erst in den letzten Jahren aufgeschlossen wurden.

6) Die Arbeit wurde 1964 abgeschlossen und für den Druck unverändert gelassen. Ihr liegen die Aufschlußverhältnisse von 1963 und 1964 zugrunde, die sich bis 1966 (in Wallertheim) und 1967 (in Sprendlingen) nicht grundlegend wandelten. 


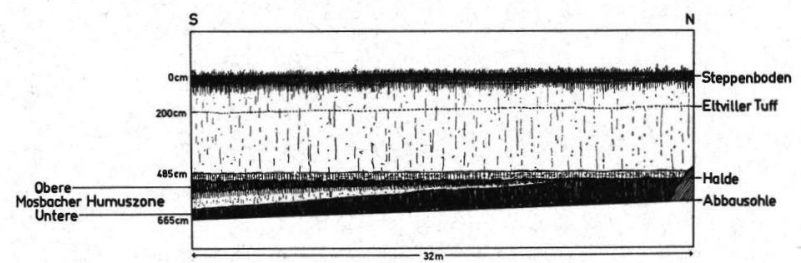

Abb. 3. Lagerung der Mosbacher Humuszonen an der Westwand in der Grube Wallertheim im Jahre 1966.

Wie schon oben angedeutet wurde, werden die beiden dunklen Böden an der Basis von Profil I und Profil II in Wallertheim nicht als gleichwertig angesehen. Gerade den unteren Boden des Profils I glaubte ZAKosek als junge Bildung ansehen zu dürfen, weil eine ${ }^{14} \mathrm{C}$ Datierung ein Alter von 28000 Jahre erbracht hat ( ${ }^{14} \mathrm{C}$-Laboratorium Heidelberg). Siehe dazu auch Leser (1967, S. 258). Die Angabe des Alters wurde mir durch Herrn Professor Dr. ZАКОSEK freundlicherweise nochmals brieflich bestätigt (1966).

Die stratigraphische Position eines Bodens dieses Alters wird aber nun von den Naßböden im Hangenden beider Profile eingenommen, die sich wiederum mit dem neuerdings in Sprendlingen aufgeschlossenen braunen Lehm parallelisieren lassen und dessen Eigenschaften mit dem "Hahnstätter (Hainerberger) Boden" von Schönhals, Rohdenburg \& Semmel (1964) übereinstimmen. In keinem Fall ist aber dieser Boden aus dem Jung/Mittelwürm-Interstadial mit den „Verbraunungszonen“ der „Erbenheimer Böden“ (= $\mathrm{E}_{1}$ bis $\mathrm{E}_{4}$ ) der o.e. Autoren zu parallelisieren, die in Wallertheim nur z. T. vorhanden, jedoch stratigraphisch durch den „Eltviller Tuff“ bestimmt sind. Im Profil Monsheim (Südrheinhessen) sind sie ebenfalls deutlich ausgebildet. Außerdem sind dort zwei der vier Horizonte mit einem Pupilla muscorum-Horizont gekoppelt, den Remy (1959) aus dem Mittelrheingebiet beschreibt. Diese typische Ausbildung fehlt dem Löß im nördlichen Teil Rheinhessens. Für die Erklärung des Vorkommens gerade in Monsheim möchte ich die unterschiedlichen Klimaverhältnisse zwischen beiden Teilen Rheinhessens heranziehen: Monsheim liegt gegenüber den regenbringenden Winden in doppelter Leelage, die eine thermische Gunst bei geringen Niederschlägen verursacht (KLUG 1961, SchweIgmanN 1967). Da im Jungwürm zur Zeit der Boden- und Lößbildung die gleichen Reliefverhältnisse geherrscht haben wie heute (LESER 1967), dürfte das auch für die reliefbedingten Ausprägungen des Klimas anzunehmen sein.

Bei Vergleich der oben gegebenen Beschreibung des neuaufgeschlossenen Unterteiles des Wallertheimer Profils (Abb. 1) mit den Angaben über den „Homburger (Erbacher) Boden“ im Sammelprofil von Schönhals, Rohdenburg \& Semmel (1964) wird deutlich, daß sich einige Übereinstimmungen der Bodenmerkmale ergeben. Zunächst stimmt die Bodenfarbe

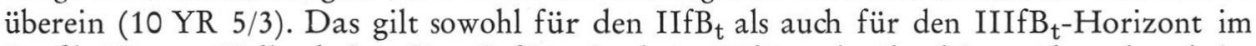
Profil II von Wallertheim. Das Gefüge ist beim IIfB $_{t}$ subpolyedrig, während es beim kompakteren IIIfB $_{\mathrm{t}}$ mehr plattig (bis polyedrig) ist. Wie beim „Homburger (Erbacher) Boden" erreicht der Tongehalt „stellenweise“ $40 \%$ :

$\begin{array}{cccc}\text { IIfB }_{\mathrm{t}} \text { (oben) } & \text { IIfB }_{\mathrm{t}} \text { (Mitte) } & \text { IIfB }_{\mathrm{t}} \text { (unten) } & \text { IIIfB }_{\mathrm{t}} \\ 36,2 \% & 38,0 \% & 37,0 \% & 39,3 \%\end{array}$

Diese Übereinstimmungen dürfen aber nicht darüber hinwegtäuschen, daß durch die stratigraphische Position im Profil und bei Vergleich mit anderen Lößprofilen unter den neuen Aufschlußverhältnissen viel eher eine Korrelierung mit den "Mosbacher Humuszonen " als mit dem eeminterglazialen Boden wahrscheinlich ist. 


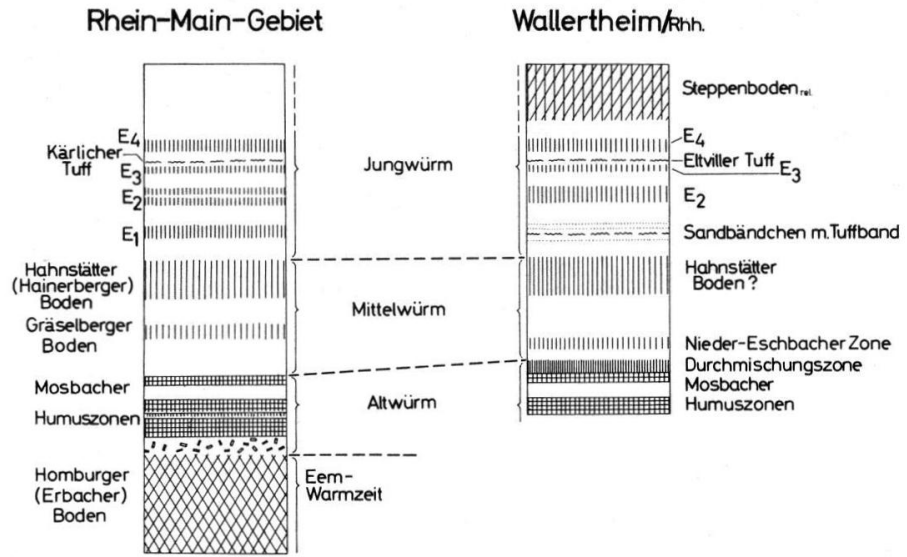

Abb. 4. Schema der Lösse, fossile Böden und Tuffe des Profils Wallertheim/Rheinhessen und des Normalprofils der Würmlößgliederung im Rhein-Main-Gebiet nach SchöNHALs, Rhodenburg u. Semmel, 1964 (unwesentlich verändert). - Die Mächtigkeit der einzelnen Straten und Horizonte beider Profile ist nicht maßstabsgleich.

Die von Schönhals, Rohdenburg \& Semmel (1964) im Sammelprofil für das RheinMain-Gebiet ausgegliederten „Mosbacher Humuszonen“ erscheinen gewöhnlich über dem „Erbacher-Boden“ als Wechselfolge von Fließerde bzw. Löß und dunklen Humuszonen (Abb. 4). Die scharfe Obergrenze der intensiver gefärbten unteren Humuszone in Wallertheim zeigt an, daß auf die Bodenbildungszeit eine Erosionsphase gefolgt sein muß. Diese erscheint auch im Normalprofil für das Rhein-Main-Gebiet und zeichnet sich durch fluviatile bis solifluidale Vorgänge aus. Die o. a. Autoren rechnen für die Folgezeit nach dem Eem mit Lessivierung und Pseudovergleyung. Im Rhein-Main-Gebiet kann für das EemInterglazial und das Altwürm wohl auch mit Steppenbodenbildung gerechnet werden. Die Lessivierung hat vermutlich den Steppenbodencharakter der eem- und nacheemzeitlichen Bodenbildungen verwischt, so daß weniger von einem $A$ - oder $B_{f^{-}}$als von einem $B_{t^{-}}$Horizont zu sprechen ist. Wie aus der Beschreibung des Sammelprofils von Schönhals, RoHDenburg \& Semmel (1964) hervorgeht, ist der Profilteil mit den „Mosbacher Humuszonen " der Abschnitt des Würm, der sich in den einzelnen Aufschlüssen ganz unterschiedlich repräsentiert findet. Die hier vorliegenden Horizonte $\operatorname{IIfB}_{t}$ und IIIfB $\mathrm{I}_{t}$ stellen in Wallertheim das Aquivalent der "Mosbacher Humuszonen“ dar. Zumindest bei der oberen Zone kann es sich sicher nicht um den Eemboden handeln, weil an ihrer Basis ein rein äolischer Löß (IIfC) auftritt, der diskordant auf dem $\operatorname{IIIfB}_{t}$ der unteren Humuszone liegt, deren Basis nicht aufschließbar war. Da für das ausgehende Eem-Interglazial trotz der Klimaverschlechterung kaum eine derart mächtige Lößakkumulation anzunehmen ist, muß dieser Profilteil einwandfrei schon in das Altwürm gestellt werden. Die Lößakkumulation muß mindestens bis auf $525 \mathrm{~cm}$ unter die rezente Landoberfläche gegangen sein, d. h. wenigstens eine $115 \mathrm{~cm}$ mächtige Lößdecke erbracht haben. Sie dürfte aber weitaus mächtiger gewesen sein, weil der IIfB $_{\mathrm{t}}$-Horizont oben deutlich gekappt ist - und zwar fluviatil. Dazu gehört dann auch der $\operatorname{IIfB}_{t} / \mathrm{C}$-Horizont, der Wirkungen der Tätigkeit fließenden Wassers erkennen läßt. Bei ihm handelt es sich um eine Durchmischungszone von Löß mit Material der hier oben liegenden „Mosbacher Humuszone“. Diese Durchmischungszone tritt im gesamten Wallertheimer Aufschluß über der oberen Humuszone auf. Nach dem Vergleich der Analysenergebnisse der Horizonte $f_{3} A(B)$ und $f_{3}(B)$ des Profils I (Leser, 1967) und den Daten von IIfB $_{t} / C$ des Profils II (Abb. 2) läßt sich $k$ e i n e Übereinstimmung feststellen. Es muß gefolgert werden, daß 1963 (LESER 1967) ein anderer Horizont aufgeschlossen gewesen ist, der sich heute in das neue Schema von Wallertheim nicht mehr einordnen läßt (siehe oben). 
Die Diskordanzen an der Oberfläche des $\operatorname{IIIfB}_{t^{-}}$, des $\operatorname{IIfB}_{t^{-}}$und des $\operatorname{IIfB}_{t} / \mathrm{C}-$ Horizontes zeigen, daß mit dem Ausgang des Eem und während des Altwürm Klimaphasen mit feuchteren, warmen oder kalten Bedingungen miteinander abwechselten. Dies nahm auch schon SchöNHALs (1958/59) an, der einen damals sichtbaren Steppenboden an der Basis des Wallertheimer Profils in das seinerzeitige Würm I (heute Altwürm) stellte. Es scheint auch ausgeschlossen, daß derartig mächtige Böden, wie sie im IIfB $_{t}-$ IIfC oder im IIIfB $_{t}$ vorliegen, nur lokale Bindungen darstellen. Trotz der von SchöNHaLs, RoHDENBURG \& SEMmel (1964) vorgeführten Vielgestaltigkeit der Altwürmbildungen ist der Schluß zulässig, daß das Profil Wallertheim in der nun hier beschriebenen Form Anschluß an die überregionale Gliederung besitzt. Der über dem $\operatorname{IIfB}_{t} / \mathrm{C}-$ Horizont auftretende Löß dürfte dann, nach der Einordnung der liegenden Horizonte in das Altwürm, in das Mittelwürm gehören, weil er im Oberteil, aber noch weit unter dem „Eltviller Tuffbändchen“, möglicherweise ein Äquivalent des „Hahnstätter (Hainerberger) Bodens" trägt.

Die Lagerung der „Mosbacher Humuszonen“ im Profil Wallertheim (Abb. 3) spricht für ein stark reliefiertes Gelände zur Zeit der Ablagerung des Löß und der Bodenbildungen. Die altwürmzeitlichen Landoberflächen haben einen etwas anderen Verlauf als heute besessen, wie aus dem Oberflächenverlauf der beiden „Mosbacher Humuszonen“ in Wallertheim hervorgeht. Sie wurden anscheinend in Mulden gebildet, die sich jedoch gegenseitig verschnitten. Deutlich wird dies am Nordende der Grubenwestwand (Abb. 3), wo beide Humuszonen zusammenfallen und einen scheinbar einheitlichen und intensiv gefärbten Boden bilden. Auch heute läßt sich noch ein Auf- und Abwandern dieser Bodenhorizonte in der gesamten Grube Wallertheim beim Nachgraben beobachten. Praktisch ab Mittelwürm, mindestens seit der Zeit, als der Löß zum Äquivalent des „Hahnstätter (Hainerberger) Bodens“ sedimentiert wurde, spielten sich Sedimentationsvorgänge und Bodenbildungen fast parallel zur rezenten Landoberfläche mit dem reliktischen postglazialen Steppenboden ab.

\section{Zur Boden- und Landschaftsentwicklung in der Würm-Kaltzeit}

Nach zahlreichen, von verschiedenen Autoren in den sechziger Jahren vorgenommenen Untersuchungen an Lößprofilen des hessischen und rheinhessischen Raumes zeichnet sich immer stärker das Bild einer klimatisch und bodengeographisch stark differenzierten Würmkaltzeit ab. Besonders SEMmel gelang es in verschiedenen Arbeiten (u. a. 1963, 1964, 1967, 1968a, b, 1969) das von Schönhals, Rohdenburg \& Semmel (1964) gegebene Normalprofil für Nordhessen und das Rhein-Main-Gebiet zu verfeinern und auszuweiten. Auch die Arbeiten von Andres (1968) und Leser (1967) konnte dazu beitragen. Gegenüber den schon fast klassischen Arbeiten von SснӧNHALs aus den fünfziger Jahren (1950, 1951, 1952, 1957a, b, 1959a, b, 1960) ergaben sich verschiedene Abweichungen.

Verfeinerung der Arbeitsmethoden und Verbesserung der Aufschlußverhältnisse führten zu der Erkenntnis, daß sich im Rhein-Main-Gebiet die Würm-Kaltzeit als eine Folge offenbar rasch wechselnder feuchter, trockener, kälterer und wärmerer Phasen repräsentiert. Diese Klimaschwankungen äußerten sich in einer großen Anzahl von fossilen Böden, Lößablagerungen und darin enthaltenen Erosionsspuren, die auf eine erhebliche Morphodynamik schließen lassen, worauf auch RohDenburg (1968) hingewiesen hat. Diese Abfolge läßt nicht mehr ohne weiteres eine schematische Gliederung der Lösse und Böden des Rhein-Main-Gebietes in Riß-Würm-Warmzeit, Alt-, Mittel- und Jungwürm zu, weil die Vielzahl der erkannten Böden und Übergangsbildungen eine Zuordnung erschweren. Auf jeden Fall dürfte aber die Berücksichtigung dieses Vorbehalts bei der Aufstellung von Regional- oder Überregionalgliederungen der Würm-Kaltzeit den tatsächlichen Verhältnissen vermehrt Rechnung tragen. 
Für das Rheinhessische Tafel- und Hügelland ergeben sich aus dem neuen Aufschluß Wallertheim (Abb. 4) und aus den o. a. jüngeren Publikationen eine Reihe Konsequenzen, die an dieser Stelle nur stichwortartig zusammengefaßt werden sollen: (1) Der letzte Interglazialboden ist in Wallertheim im Augenblick nicht bestimmbar. Möglicherweise liegt er in einem unter starkem Wassereinfluß gebildeten Boden vor, der an einer tiefen Stelle der Grube aufgeschlossen ist und wo auch die paläolithische Jägerstation (SCHMIDTGEN \& Wagner 1929, 1931) gefunden worden sein soll. - Das Profil wurde von mir am 5. 8. 1963 aufgenommen (Feld-Nr. 444, Wallertheim III), jedoch nicht publiziert. Korngrößenanalysen und verschiedene andere Untersuchungsdaten liegen ebenfalls vor. Der seinerzeit postulierte verbraunte Steppenbodenrest (LESER 1967), der aus einer Schwarzerde hervorgegangen ist, konnte nicht wieder aufgefunden werden. Daß in Rheinhessen Schwarzerdebildung während des Eem möglich oder auch $\mathrm{n}$ icht möglich gewesen ist, bedarf noch der Beweise. (2) Mindestens zwei der "Mosbacher Humuszonen“ liegen in Wallertheim vor. Sie deuten, weil auf rein äolischem und nicht ungelagerten Löß entwickelt, auf starke Klimaschwankungen im ersten Abschnitt des Würm hin. (3) Der folgende Teil des Lößpaketes enthält vermutlich die Nieder-Eschbacher Zone (Naßboden) sowie als zweiten mächtigeren Boden eine Bildung, die vielleicht das Paudorf-Interstadial markieren könnte. Der zuletzt erwähnte Boden tritt in mehreren rheinhessischen Profilen auf. In Sprendlingen ging er in einen braunen mächtigen Boden über. (4) Der letzte Abschnitt der WürmKaltzeit ist wiederum durch eine Reihe leichter Klimaschwankungen gekennzeichnet, die sich in Lößumlagerung, Sandbändchen- und Naßbodenbildung äußern. Den zwei (drei?) Naßböden in Wallertheim entsprechen zahlreiche weitere solche Böden vom Typ des Frosttundrengleys in vielen Lößprofilen des Rheinhessischen Tafel- und Hügellandes. (5) Der stratigraphische Wert des Tuffbändchens hat gegenüber den fünfziger Jahren eingebüßt,

\section{Literatur}

ANDREs, W.: Beobachtungen zur Gliederung eines Würmprofiles und zur spätwürmzeitlichen und holozänen Hangüberformung bei Marienborn (Rheinhessen). - Mainzer Naturwiss. Arch., 7, 131-140, Mainz 1968.

BrunnaCker, K.: Zur Parallelisierung des Jungpleistozäns. - Geol. Jb., 76, 129-150, Hannover 1958.

FalKe, H.: Rheinhessen und die Umgebung von Mainz. - Sammlg. Geol. Führer, Bd. 38, 156 S., Berlin 1960.

FAuler, W.: Das Deckschichtenprofil und das geologische Alter der Moustierstation von Wallertheim (Rheinhessen). - Jahresber. u. Mitt. Oberrhein. geol. Ver., N.F., 27, 116-140, Stuttgart 1938.

Frechen, J.: Die Tuffe des Laacher Vulkangebietes als quartärgeologische Leitgesteine und Zeitmarken. - Fortschr. Geol. Rheinland u. Westf., 4, 363-370, Krefeld 1959 (1959a).

- : Die basaltischen "Ausbläser" von Kärlich (Neuwieder Becken) und die Verbreitung ihrer Tuffe. - Fortschr. Geol. Rheinland u. Westf., 4, 301-312, Krefeld 1959 (1959b).

KLUG, H.: Das Klima Rheinhessens in seiner kleinräumigen Gliederung. - Mitteilungsbl. rheinh. Landeskde., 10, 321-327, Wörrstadt 1961.

Leser, H.: Geomorphologische Gliederung des Alzeyer Hügellandes. - Mitteilungsbl. rheinh. Landeskde., 14, 224-231 und 238-241, Wörrstadt 1965.

- : Geomorphologische Ubersichtskarte des Rheinhessischen Hügellandes. Geomorphologische Einheiten und Gliederung einer oberrheinischen Landschaft. - Ber. z. dt. Landeskunde, Br. 36, 65-88, Bad Godesberg 1966.

- : Beobachtungen und Studien zur quartären Landschaftsentwicklung des Pfrimmgebietes (Südrheinhessen). - Arbeiten z. rhein. Landeskunde, H. 24, 442 S., Bonn 1967.

RemY, H.: Zur Gliederung des Lösses bei Kärlich und Bröl am unteren Mittelrhein mit besonderer Berücksichtigung der Faunen. - Fortschr. Geol. Rheinland u. Westfalen, 4, 323-330, Krefeld 1969.

Rohdenburg, H.: Jungpleistozäne Hangformung in Mitteleuropa - Beiträge zur Kenntnis, Deutung und Bedeutung ihrer räumlichen und zeitlichen Differenzierung. - Gött. Bodenkdl. Ber., 6, 3-107, Göttingen 1968.

Schmidtgen, O. u. WAGNer, W.: Eine altpaläolithische Jagdstelle bei Wallertheim in Rheinhessen. Notizbl. Ver. f. Erdkde. u. hess. geol. L.-Anst. f. 1928, V. F., H. 11, 59-89, Darmstadt 1929. 
Schmidtgen, O. u. WAgner, W.: Diluvium und Tertiär im Innern des Mainzer Beckens (Bericht über die Begehungen der Hauptversammlung in Mainz). - Z. deutsch. geol. Ges., 83, 687, Stuttgart 1931.

ScнöнaLs, E.: Uber einige wichtige Lößprofile und begrabene Böden im Rheingau. - Notizbl. hess. L.-Amt Bodenforsch., (VI) 1, 244-259, Wiesbaden 1950.

- : Fossile gleiartige Böden des Pleistozäns im Usinger Becken und am Rand des Vogelsberg. Notizbl. hess. L.-Amt Bodenforsch., (VI) 2, 160-183, Wiesbaden 1951.

- : Ergebnisse neuer Untersuchungen an Lößböden des Vogelsberg und seiner Randgebiete. Notizbl. hess. L.-Amt Bodenforsch., (VI) 3, 307-340, Wiesbaden 1952.

- : Eine äolische Ablagerung der jüngeren Tundrenzeit im Habichtswald. - Notizbl. hess. L.Amt Bodenforsch., 85, 380-386, Wiesbaden 1957 (1957a).

- : Lößstratigraphie und fossile Böden des Mittel- und Jungpleistozäns in Hessen. - INQUA, 5. Congr. Int., Rés. des communications, 173-174, Madrid-Barcelona 1957 (1957b).

- : Der Basalt-Tuff von Kärlich als Leithorizont des Würm-Hochglazials. - Fortschr. Geol. Rheinland u. Westf., 4, 313-322, Krefeld 1959 (1959a).

- : Klima, äolische Sedimentation und Bodenbildung während der Eiszeit in Hessen. - Z. deutsch. geol. Ges., 111, 251-253, Hannover 1959 (1959b).

- : Spät- und nacheiszeitliche Entwicklungsstadien von Böden aus äolischen Sedimenten in Westdeutschland. - Proc. 7. Int. Congr. Soil Sci. Madison USA, 283-290, Madison, Wisc., USA 1960.

Schönhals, E.: Rohdenburg, H. u. Semmel, A.: Ergebnisse neuerer Untersuchungen zur Würmlöß-Gliederung in Hessen. - Eiszeitalter u. Gegenwart, 15, 199-206, Öhringen 1964.

Schweigmann, F.: Die Niederschlagsverhältnisse im Raume Worms, aufgezeigt an Meßergebnissen neueren Datums. - Mitteilungsbl. rheinh. Landeskde., 16, 327-334, Wörrstadt 1967.

Semmel, A.: Mitteilungen über ein Pleistozänprofil bei Hahnstätten (Limburger Becken). - Notizbl. hess. L.-Amt f. Bodenforsch., 91, 359-365, Wiesbaden 1963.

- : Beobachtungen zur Gliederung pleistozäner Sedimente im Raum Flörsheim-Kriftel-Kelsterbach. - Z. deutsch. geol. Ges., 116, 985-986, Hannover 1964.

- : Neue Fundstellen von vulkanischem Material in hessischen Lössen. - Notizbl. hess. L.-Amt Bodenforsch., 95, 104-108, Wiesbaden 1967.

- : Studien über den Verlauf jungpleistozäner Formung in Hessen. - Frankf. Geogr. Hefte, Nr. 45, 133 S., Frankfurt 1968 (1968a).

— : Die Lößdecke im Dyckerhoff-Steinbruch bei Wiesbaden-Biebrich. - Mainzer Naturw. Arch., 7, 74-79, Mainz 1968 (1968a).

- : Bemerkungen zur Würmlößgliederung im Rhein-Main-Gebiet. - Notizbl. hess. L.-Amt Bodenforsch., 97, Wiesbaden 1969.

WaGNER, W.: Geologische Karte von Hessen $1: 25$ 000. Hrsg. v. d. Hess. Geol. L.-Anst., Blatt 6114 Wörrstadt, Darmstadt 1935.

Woldstedt, P.: Das Eiszeitalter. Grundlinien einer Geologie des Quartärs. 3 Bde., Stuttgart $1958-1965$.

Zaкоseк, H.: Zur Genese und Gliederung der Steppenböden im nördlichen Oberrheintal. - Abh. hess. L.-Amt Bodenforsch., H. 37, 46 S., Wiesbaden 1962.

Manuskr. eingeg. 17. 10. 1969.

Anschrift des Verf.: Dozent Dr. rer. nat. H. Leser, Geogr. Institut der Technischen Universität Hannover. 3 Hannover, Im Moore 21. 